\title{
Behavioral patterns of Djungarian hamsters: An adaptive profile
}

\author{
D. KIM SAWREY, DENIS J. BAUMGARDNER, MICHAEL J. CAMPA, BRUCE FERGUSON, \\ ALAN W. HODGES, and DONALD A. DEWSBURY \\ University of Florida, Gainesville, Florida
}

\begin{abstract}
Djungarian hamsters (Phodopus sungorus) were observed in a series of laboratory tests, including home cage activity, wheel running, open-field behavior, sexual dimorphism for body mass, copulatory behavior, tonic and dorsal immobility, climbing, digging, nest building, and parental behavior. Fourteen resulting measures were compared with previous results from this laboratory from a variety of muroid species. A cluster of behavioral adaptations emerged with several marked similarities to the behaviors of a group of North American Microtus species, whereas other behaviors appeared to more closely reflect the classification of the hamsters as cricetines. It is suggested that Phodopus and Micronus species may have evolved many similar adaptive characteristics in response to shared environmental variables.
\end{abstract}

The description and understanding of the behavioral adaptations of organisms are tasks of major importance confronting students of animal behavior. With comparisons of a number of behavior patterns in a variety of species, clusters of behavioral adaptations emerge. These associations of behavioral adaptations, coupled with information regarding the ecological, morphological, and physiological attributes of members of related species, will lead to testable hypotheses concerning the relations among these variables. The resulting general laws may describe a complex of adaptations that are applicable across a wide selection of taxa (Jarman, 1982).

Rodents of the superfamily Muroidea are numerous and widely distributed; they inhabit a great range of ecological niches, making them particularly valuable as subjects in comparative research. Most of these species are small, nocturnal, and generally secretive in their habits, making field observations of behavior difficult. However, laboratory investigations can allow direct comparisons of several species in a variety of standardized testing situations. In addition, laboratory tests can be selected that allow behaviors to be quantified easily and data to be collected quickly.

Over the past 15 years, muroid rodents have been the subjects of a substantial behavioral research program in this laboratory. Data for from 6 to 42 species are available for home cage activity (Baumgardner, Ward, \& Dewsbury, 1980 ), wheel running (Dewsbury, 1980), open-field behavior (Webster, Baumgardner, \& Dewsbury, 1979; Wilson, Vacek, Lanier, \& Dewsbury, 1976), sexual dimorphism for body mass (Dewsbury, Baumgardner, Evans, \& Webster, 1980; Dewsbury \& Dawson, 1979), copulatory

This research was supported by Grant BNS82-00689 from the National Science Foundation. We thank Michael R. Murphy for providing breeding stock. Please address requests for reprints to D. A. Dewsbury, Department of Psychology, University of Florida, Gainesville, FL 32611. behavior (e.g., Dewsbury, 1975), tonic and dorsal immobility responses (Webster, Lanthorn, Dewsbury, \& Meyer, 1981), climbing (Dewsbury, Lanier, \& Miglietta, 1980), digging (Webster, Williams, Owens, Geiger, \& Dewsbury, 1981), nest building (Hartung \& Dewsbury, 1979a), and parental behavior (Hartung \& Dewsbury, 1979b), among others. This body of data allows comparison of a number of species on a number of behavioral tasks, all conducted in the same laboratory. The pattern of scores of an individual species can be identified, resulting in what we have called the "adaptive profile" (Dewsbury, Baumgardner, Sawrey, \& Webster, 1982) of that species.

The subjects of the present work, Phodopus sungorus campbelli, were tested on each of the above 10 tasks. Hamsters of the genus Phodopus inhabit much of central Asia. The range of $P$. s. campbelli extends through the steppes and semiarid regions of central Asia "from the alpine steppes of the Altai, Tuva, and Mongolia to Transbaikalia and Manchuria" (Vorontsov, Radzhabli, \& Lyapunova, 1967). They share with other true hamsters short legs, a short tail, and a rather squat appearance. They also possess characteristic buccal pouches. Phodopus are omnivorous and semifossorial (Flint, 1966).

Phodopus sungorus campbelli are being used increasingly in behavioral and physiological research (e.g., Daly, 1976; Hoffman, 1982), but little is known of their behavior in the field or in relation to that of other species. The present research represents an attempt to compare the behavioral patterns of $P$. s. campbelli with those of other species and to provide a framework for future behavioral research.

\section{GENERAL METHOD}

All subjects were Djungarian hamsters, Phodopus sungorus, and were maintained in a windowless air-conditioned room with Purina laboratory animal chow and water available ad lib. The colony room was kept on a reversed 16:8 photoperiod with light onset at $1730 \mathrm{~h}$. Unless otherwise 
specified, the experiments were conducted during the dark phase. The age ranges of the animals used in each study were matched as closely as was practical to the ages of the subjects of similar experiments conducted previously in this laboratory. Where not specifically noted, the animals were housed individually in $29 \times 19 \times 13 \mathrm{~cm}$ clear plastic cages prior to testing. Exclusive of the animals used to collect dimorphism data, 31 females each appeared in one study per individual and 65 males appeared in from one to three studies per individual.

\section{EXPERIMENT 1}

The purpose of the first experiment was to investigate the distribution of the behavioral patterns of $P$. sungorus across a 24-h period. Recently Baumgardner et al. (1980) related diurnal activity patterning in several muroid rodent species to their respective food requirements, basal metabolic rates, and various environmental factors, using the same experimental methods.

\section{Method}

Twelve adult (85-180-day-old) males were placed individually in 29 $\times 19 \times 13 \mathrm{~cm}$ clear plastic cages $60 \mathrm{~h}$ prior to testing. San-i-cel was provided as substrate. Behavioral observations were recorded during alternate hours throughout a single 24-h period. The behavior of each of the 12 animals was noted on a checklist every $60 \mathrm{sec}$, using an instantaneous sampling procedure as described by Altmann (1974). One of the following mutually exclusive behavioral patterns was recorded for each animal once every $60 \mathrm{sec}$ : locomotion, eating, grooming, inactivity, drinking, stereotypy, postural readjustment, or sleep. Additional procedural details were those used by Baumgardner et al. (1980).

\section{Results and Discussion}

The distribution of eight behavioral patterns displayed during the dark phase, the light phase, and over the entire 24-h period is presented in Table 1 . Sleep was the most commonly displayed behavior, accounting for $69 \%$ of all observations. This value is the highest recorded among the 16 species previously tested in this laboratory under similar conditions. Levels of locomotion, stereotypy, and inactivity were very low compared to the values obtained for previously investigated species. Nocturnal ratios, the ratio of the percentage of time a given behavioral activity is observed in the dark phase divided by the percentage of time the behavior is observed in the light phase, are presented for each behavior. The percentages for most behaviors were very similar in the light and in the dark.
The nocturnal ratio for stereotypy suggests a strong nocturnality for that pattern. However, the total number of instances of the behavior was low ( 24 of 780 observations) and two individual hamsters accounted for 23 of $24 \mathrm{oc}-$ currences.

Baumgardner et al. (1980) separated behaviors into two distinct groups: composite activity and composite inactivity. Composite activity, a measure that includes locomotion, stereotypy, grooming, eating, and drinking, remained at fairly constant levels throughout the testing period (Figure 1). Thus, $P$. sungorus are basically acyclic rather than nocturnal with respect to the patterning of behavior in the present testing situation.

\section{EXPERIMENT 2}

In the second experiment, we investigated another measure of daily activity: wheel running. In this laboratory, the wheel running of 15 other rodent species had been examined previously under similar conditions. In addition, other investigators have collected data for other species using similar techniques, thereby providing ample opportunity for species level comparisons.

\section{Method}

Twelve male (60-90 days) $P$. sungorus were placed singly in Wahmann LC-34 running wheels for a period of 29 days. For the final 14 days, Stoelting printout counters recorded the number of revolutions made every hour by each individual.

\section{Results and Discussion}

The 12 hamsters in this experiment made a mean of 627 revolutions per day, the lowest total for any species recorded in this laboratory. Three of the animals made considerably more revolutions per day (mean $=1,616.5$ ) than the remaining 9 animals (mean $=297.1$ ). Additionally, these 3 animals showed a strong nocturnal tendency in their running, which contrasts sharply with the acyclic patterns of running shown by the other animals (see Figure 2). When the means for all 12 animals were considered, $P$. sungorus appear to be nocturnal in their pattern of wheel running, making a mean of 479.7 revolutions per day in the dark phase and 147.2 revolutions per day in the light phase.

Table 1

Summary of the Percentages of the Dark Phase, Light Phase, and Total Day Spent in Eight Categories of Behavior

\begin{tabular}{|c|c|c|c|}
\hline Behavior & Dark & Light & $\begin{array}{c}\text { Nocturnal Ratio } \\
\text { (Dark/Light) }\end{array}$ \\
\hline $\begin{array}{l}\text { Locomotion } \\
\text { Stereotypy } \\
\text { Grooming } \\
\text { Eating } \\
\text { Drinking } \\
\text { Sleep } \\
\text { Postural Readjustment } \\
\text { Inactivity }\end{array}$ & $\begin{array}{r}4.20 \\
.76 \\
13.82 \\
10.83 \\
.21 \\
65.69 \\
2.95 \\
1.53\end{array}$ & $\begin{array}{r}5.52 \\
.03 \\
10.26 \\
9.38 \\
.30 \\
71.35 \\
2.00 \\
1.16\end{array}$ & $\begin{array}{r}.76 \\
25.33 \\
1.35 \\
1.15 \\
.70 \\
.92 \\
1.48 \\
1.32\end{array}$ \\
\hline $\begin{array}{l}\text { Composite Activity } \\
\text { Composite Inactivity }\end{array}$ & $\begin{array}{l}29.82 \\
68.64\end{array}$ & $\begin{array}{l}25.49 \\
73.35\end{array}$ & $\begin{array}{r}1.17 \\
.94\end{array}$ \\
\hline
\end{tabular}




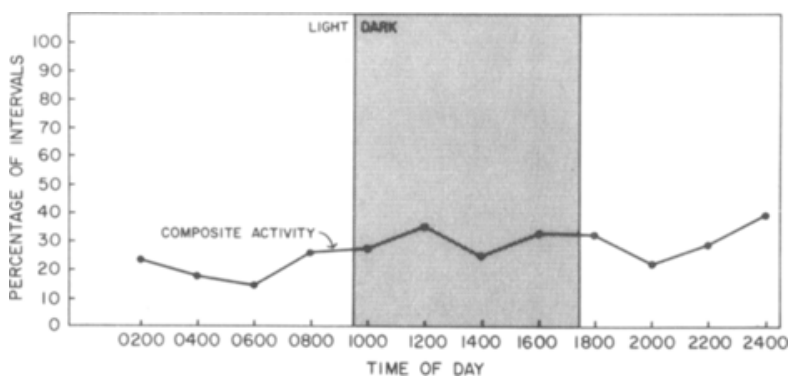

Figure 1. Hour-by-hour summary of the patterning of composite home cage activity relative to photoperiod in Phodopus sungorus.

\section{EXPERIMENT 3}

Various open-field apparatus have been used to measure behavioral tendencies and activity levels in a wide variety of animal taxa. The 20 species of wild rodents for which we have collected data provide a basis for comparison of several open-field measures of potential interest to an individual investigator.

\section{Method}

The 10 male hamsters (90-180 days) used in this experiment were housed individually for at least 2 weeks prior to testing. The open-field apparatus was constructed of plywood and measured $101.6 \times 101.6 \times 45.7 \mathrm{~cm}$. It was painted flat gray with black lines on the floor demarcating 25 equalsize squares. Each 10-min test was initiated by the placement of an animal in a corner square. One observer recorded the number of square crossings and type of square entered using a set of microswitches connected to a printout counter. Square type was defined by the number of walls bounding a square: two walls ( $2 W$ ), one wall (IW), and zero wall (OW). Utilizing an Esterline-Angus operations recorder, the following mutually exclusive behaviors were recorded by a second observer: rearing, rearing at wall, freezing, gnawing at the open field, jumping, locomotorexploratory behavior, grooming, head movement, and climbing. Illumination was provided by a single $25-\mathrm{W}$ red light suspended over the center of the apparatus. At the end of each test, the number of boluses in the open field was recorded. The open field was cleaned before each test with a pine-scented detergent. Additional procedural details were those used by Wilson et al. (1976).

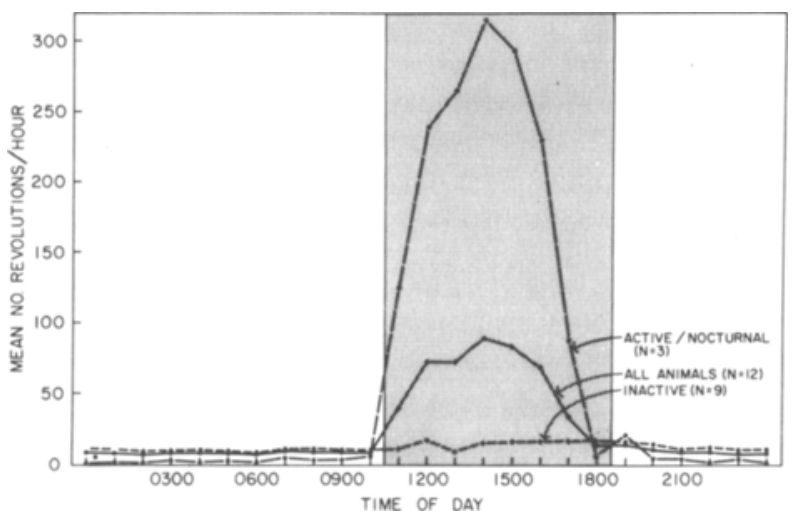

Figure 2. Hour-by-hour summary of the patterning of wheel running relative to photoperiod in Phodopus sungorus.

\section{Results and Discussion}

Hamsters entered a mean of 283.4 squares and deposited a mean of 3.8 boluses per test. The numbers of squares entered in each successive 2 -min period were $86.6,66.5$, 46.6, 41.3, and 42.4. A repeated measures analysis of variance revealed a significant difference in square entries by period $[F(4,36)=8.32, p<.001]$. Utilizing a Newman-Keuls test, significant differences were found between Period 1 and each of the last three periods. No other comparisons were significant.

The animals made a mean of 14.4 entries per available IW square, 14.0 entries per available $2 W$ square, and 6.0 entries per available OW square. An analysis of variance indicated that there was a significant effect on number of entries as a function of square type $[F(2,27)=7.35$, $\mathrm{p}<.01$ ]. A Newman-Keuls test confirmed that significantly fewer entries were made into $0 \mathrm{~W}$ squares than into IW or $2 \mathrm{~W}$ squares.

The percentages of time spent in each of the behavioral categories were as follows: rearing, 3.4; rearing at wall, 10.4; freezing, 0.6; gnawing at the open field, 0.7; scratching the open field, 7.4; jumping, 0.3; locomotorexploratory behavior, 49.8; grooming, 21.7 ; head movement, 0.1 ; climbing, 0.1 . The score for scratching the open field was the highest yet recorded in this laboratory. The level of grooming was among the highest we have recorded, while the percentage of time spent moving only the head was among the lowest.

\section{EXPERIMENT 4}

It has been suggested by several authors that the extent of sexual dimorphism within a species is correlated with the mating system of the species (Kleiman, 1977; Ralls, 1976). Specifically, sexual dimorphism should be greatest when one sex competes for access to the other; among mammals, this most commonly takes the form of malemale interactions favoring larger males. This experiment measured sexual dimorphism for body mass for $P$. sungorus.

\section{Method}

Animals were housed as complete litters in either $29 \times 19 \times 13 \mathrm{~cm}$ or $48 \times 27 \times 13 \mathrm{~cm}$ plastic cages, depending on the size of the litter. Only litters that included at least one male and one female were used for analysis. All animals were weighed on an Ohaus Model 1630 animal balance at 35 and 90 days of age. The mean mass of males and the mean mass of females in each of 25 litters were used as the basic data for analysis.

\section{Results and Discussion}

Mean body mass for males was significantly greater than that for females at both 35 and 90 days of age. Because Ralls (1976) has suggested that the ratios of the cube roots of body masses are more accurate estimates of body length ratios than are mass ratios themselves, cube root ratios are also provided (Table 2 ). 
Table 2

Data Relating to Sexual Dimorphism for Body Mass

\begin{tabular}{|c|c|c|}
\hline Measure & Day 35 & Day 90 \\
\hline No, of Litters & 25 & 25 \\
\hline No. of Males & 44 & 42 \\
\hline No. of Females & 51 & 52 \\
\hline M Body Mass Males (SD) & $26.5 \quad(5.0)$ & 31.1 \\
\hline M Body Mass Females (SD) & $23.8 \quad(4.5)$ & $27.3 \quad(2.4)$ \\
\hline Male vs. Female $t$ & 4.09 & 5.19 \\
\hline $\mathrm{p}$ & .001 & .001 \\
\hline Cube Root Ratio & 97 & 96 \\
\hline
\end{tabular}

Note-Body mass is expressed in grams. Within-subject $t$ is reported.

\section{EXPERIMENT 5}

The copulatory patterns of rodent species vary both quantitatively and qualitatively. Utilizing a comparative approach, Dewsbury $(1975,1978)$ has attempted to determine the adaptive significance of the various observed copulatory patterns. The copulatory behavior of $P$. sungorus was investigated in an effort to identify the pattern typical of the species and elucidate any parameter that might provide a clue to their social structure.

\section{Method}

Copulatory tests were conducted in the $48 \times 27 \times$ $13 \mathrm{~cm}$ plastic home cages of the males. One hour was allowed for the initiation of copulation. Tests in which copulation did not occur were not included in the analyses. Tests were terminated after a satiety criterion of $30 \mathrm{~min}$ without copulation. Females were brought into behavioral estrus with an injection of $.06 \mathrm{mg}$ estradiol benzoate $72 \mathrm{~h}$ prior to testing and an injection of $.6 \mathrm{mg}$ progesterone 6-8 $\mathrm{h}$ prior to testing.

Eleven male-female pairs (110-200 days) were observed in 37 tests in which copulation occurred. All pairs were observed in at least two tests; 10 pairs were observed in three tests and 5 pairs were observed in four tests.

The copulatory pattern of $P$. sungorus is characterized by groups or "series" of mounts (male mounts female without vaginal penetration), intromissions (male mounts and achieves vaginal penetration), and ejaculation (intromissions with ejaculations). Ejaculations were easily identifiable on the basis of behavioral criteria (see below) that were validated with vaginal smears. Standard measures of copulatory behavior were recorded, including mount latency $(\mathrm{ML}$, the time from introduction of the female to the first mount or intromission), intromission latency (IL, the time from introduction of the female to the first intromission), ejaculation latency ( $\mathrm{EL}$, the time from the first intromission of a series to ejaculation), ejaculation frequency (EF, the number of ejaculations per satiety test), intromission frequency (IF, the number of intromissions to ejaculation), mount frequency (MF, the number of mounts preceding ejaculation), mean interintromission interval (MIII, the mean interval between intromissions of a series), and postejaculatory interval (PEI, the time from ejaculation to the first intromission of the next series).

\section{Results and Discussion}

Phodopus sungorus pairs typically display a single brief intravaginal thrust with each insertion, multiple intromissions prerequisite to ejaculation, and multiple ejaculations in an individual session. Very occasionally, usually in a later series, a male will thrust more than once during a single insertion. This aspect of the copulatory pattern is similar to that seen in golden hamsters, the only other species in which it has been observed. It has previously been reported that $\boldsymbol{P}$. sungorus display a mechanical tie between the penis and vagina (a lock) at ejaculation (Daly, 1976; Emmerick, 1978a, 1978b). However, never during any of the approximately 100 ejaculations observed for this and other studies did we observe definitive evidence of a lock. At ejaculation, the pair typically fell to one side, the female firmly grasped by the forepaws of the male. The pair remained in this position for a mean of $9.7 \mathrm{sec}$. In no instance was the female able to extricate herself from the grasp of the male, and thus the existence of a lock was decisively demonstrated. In addition, repeated viewing of motion picture film of several ejaculations did not produce any observable evidence of a physical tie between penis and vagina. Thus, it appears either that there is no lock and proximity at ejaculation is due to the grasp of the male or that the duration of the lock coincides exactly with the duration of the clasp of the male. However, since Emmerick (1978a, 1978b) reported the duration of the lock as $10 \mathrm{sec}$, it seems reasonable to assume that we are measuring the same behavior.

Phodopus sungorus pairs attained a mean of 2.9 ejaculations per test. Mount and intromission latencies were $1,221.9$ and $1,894.7 \mathrm{sec}$, respectively. Other standard measures of copulatory behavior are presented in Table 3 . A copulatory plug is deposited at ejaculation.

\section{EXPERIMENT 6}

There has recently been a sizable increase in the number of investigations of various complex inhibitory responses such as the tonic immobility response. Research has focused on the possible adaptive significance of such responses (e.g., Gallup, 1977) as well as their physiological bases (Hatton, Lanthorn, Webster, \& Meyer, 1978; Klemm, 1971). In a previous comparative study of two im- 
Table 3

Measures of Copulatory Behavior in Phodopus sungorus

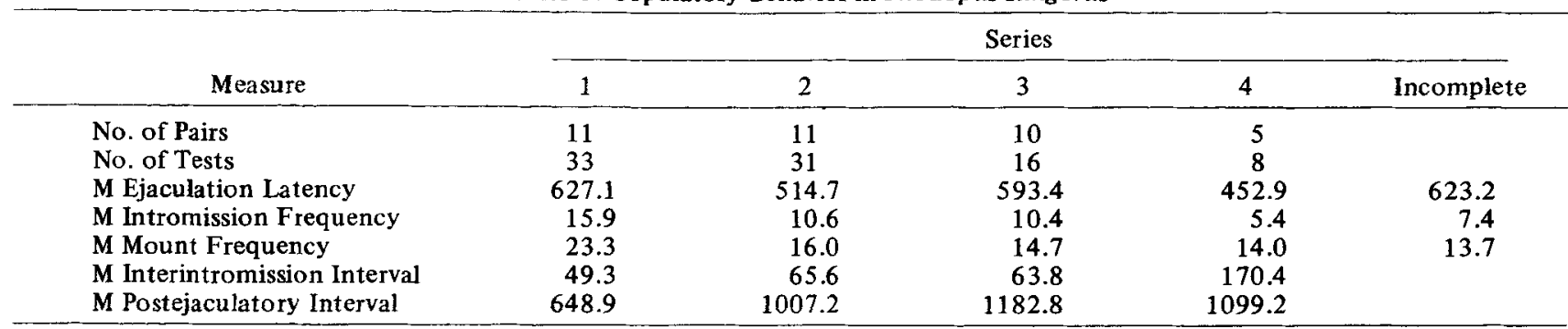

Note-Latencies and intervals are expressed in seconds.

mobility responses (Webster, Lanthorn, et al., 1981), tonic immobility and the dorsal immobility response, both phylogenetic affinities and effects of environmental variables emerged.

\section{Method}

The two immobility responses were studied in 10 male hamsters $(70-110$ days) using the procedures of Webster, Lanthorn, et al. (1981). The duration of immobility was measured for each animal for three trials each of tonic and dorsal immobility. All animals received three trials for one type of immobility followed at least $1 \mathrm{~h}$ later by three trials for the second type of immobility. Trials of like type were separated by $15 \mathrm{sec}$. The order of testing was counterbalanced. Trials were terminated after a maximum immobility duration of $300 \mathrm{sec}$.

Tonic immobility was induced by rapidly inverting an animal in a Vshaped wooden trough $(18 \times 9 \times 3.5 \mathrm{~cm})$. Gentle pressure was maintained until the animal ceased struggling or for a maximum of $10 \mathrm{sec}$, at which point the trial was terminated and scored as negative. The dorsal immobility response was induced by simply lifting the animal off its feet by the scruff of the neck. Again, the trial was terminated if the response was not induced within $10 \mathrm{sec}$.

\section{Results and Discussion}

The hamsters responded on only 2 of 30 trials of tonic immobility but on 29 of 30 trials of dorsal immobility (see Table 4). A single animal accounted for both positive tonic immobility trials. The mean durations for both responses were low in comparison with the other 13 species previously investigated in this laboratory. The mean duration for tonic immobility, when all trials were considered, was the lowest yet observed.

\section{EXPERIMENT 7}

The environments inhabited by various rodent species offer varying opportunities for climbing. Several species possess adaptations for a largely arboreal existence, while others are mainly terrestrial or fossorial. Experiment 7 was designed to provide an objective measure of the tendency of $P$. sungorus to climb.

\section{Method}

Climbing was studied using the procedures of Dewsbury, Lanier, and Miglietta (1980). The apparatus consisted of a $1 / 4$-in. hardware-cloth cone, with a diameter of $28 \mathrm{~cm}$ at its base and a height of $55 \mathrm{~cm}$. The top of the cone bisected a 93-cm dowel rod onto which animals were free to climb. The entire apparatus was placed in a large wooden box with a Plexiglas front to permit viewing. The animals were placed singly into the box, and all instances of climbing on the cone and rod recorded for $5 \mathrm{~min}$.

\section{Results and Discussion}

None of the 10 hamsters climbed in this apparatus; no hamster left the ground. All 12 previously tested species had spent at least some time off the ground.

\section{EXPERIMENT 8}

Digging is a behavior that is typically very readily elicited in the laboratory and is presumably related to several aspects of the ecology of a species. In a previous study in this laboratory, the digging behavior of 12 taxa of muroid rodents was reported (Webster, Williams, et al., 1981).

\section{Method}

Twenty male $P$. sungorus were used in this experiment. Animals were placed individually in a $61.0 \times 58.0 \times 59.5 \mathrm{~cm}$ plywood box with a Plexiglas front to permit observation. A single $25-W$ red light bulb, suspended through the ceiling, provided illumination. Half of the animals were tested on a sand substrate and half on a peat substrate. The depth of both substrates was approximately $5 \mathrm{~cm}$. Each hamster was observed on 2 consecutive days in trials of $10 \mathrm{~min}$ each.

Table 4

Results of the Study of Tonic and Dorsal Immobility

\begin{tabular}{lccc}
\multicolumn{1}{c}{ Measure } & $\begin{array}{c}\text { Tonic } \\
\text { Immobility }\end{array}$ & $\begin{array}{c}\text { Dorsal } \\
\text { Immobility }\end{array}$ & \multicolumn{1}{c}{ Statistics } \\
\hline No. Hamsters Responding & $1 / 10$ & $10 / 10$ & $\chi^{2}=7.36, \mathrm{p}<.01$ \\
M No. Trials with Response & .2 & 2.9 & $\mathrm{~T}=0, \mathrm{p}<.01$ \\
M Duration of all Trials & 2.2 & 29.0 & $\mathrm{~T}=0, \mathrm{p}<.01$ \\
M Duration of Trials with Response Only & 33.0 & 30.0 & \\
\hline
\end{tabular}

Note $-T$ values are by Wilcoxon matched-pairs, signed-ranks tests. Durations are expressed in seconds. 
Table 5

Results of Digging Study

\begin{tabular}{lrrr}
\multicolumn{1}{c}{ Measure } & Sand & Peat \\
\hline Percent Hamsters Digging & 100 & 70 \\
Percent Trials With Digging & 75 & 50 \\
M Duration Digging (Digging Animals Only) & 14.1 & 26.4 \\
M Number Bouts Digging (Digging Animals Only) & 10.6 & 6.1 \\
M Duration Digging (All Animals) & 3.7 & 13.2 \\
M Number Bouts Digging (All Animals) & 3.1 \\
\hline
\end{tabular}

Note-Durations are expressed in seconds.

Bouts of digging were recorded using an Esterline-Angus operations recorder. Two additional measures, latency to dig and number of bouts of digging, were subsequently extracted from the record. A bout of digging was defined as an unbroken interval of digging. Animals that did not dig were assigned a latency of $600 \mathrm{sec}$.

\section{Results and Discussion}

The hamsters spent a mean of $10.6 \mathrm{sec}$ per day digging on sand and a mean of 13.2 sec digging on peat. The mean time spent digging did not differ by day (Wilcoxon $\mathrm{T}=$ $66.5, \mathrm{p}>.05$ ) or by substrate (Mann-Whitney $U=40$, $\mathrm{p}>.05$ ). All animals dug on at least 1 day on sand, whereas only 7 of 10 dug on peat. Table 5 shows mean latencies to dig, durations of digging, and number of bouts on both substrates.

\section{EXPERIMENT 9}

Nest building is widespread among muroid rodents. Some species build very simple nests or simply utilize the available substrate unaltered, whereas other species build large and elaborate nests. Various factors are presumed to affect the size and type of nest built: ambient temperature, metabolism, available materials, etc. In Experiment 9, we measured both amount of nesting material used and type of nest constructed in a standard laboratory testing situation.

\section{Method}

Ten males and 10 females (50-70 days of age at the beginning of data collection) individually housed in $29 \times 19 \times 13 \mathrm{~cm}$ clear plastic cages were used as subjects. Unbleached cotton batting was placed in the wiremesh food hopper of the cage lid for 3 days prior to the beginning of data collection. Data were then collected for 14 consecutive days. Each day, the lid and cotton batting were weighed to determine the amount of cotton pulled into the cage by the animal. The cotton in the cage was removed daily, and the supply in the food hopper was supplemented as needed.

Ratings of nest type were made daily using the following scale: 0 , no nest constructed; 1 , a pallet of cotton had been formed but it lacked sides and a cover (platform nest); 2 , a bowl- or cup-shaped nest had been constructed but it lacked a cover (cup-shaped nest); and 3, a bowl- or cupshaped nest had been constructed and a cover was present (covered nest).

Purina laboratory rodent chow was available ad lib inside the cage, and San-i-cel was used as substrate. Additional procedural details were as used by Hartung and Dewsbury (1979a).

\section{Results and Discussion}

Males pulled a mean of $2.3 \mathrm{~g}$ and females a mean of $1.9 \mathrm{~g}$ of cotton per day. The sex difference was not significant (Mann-Whitney $\mathrm{U}=26, \mathrm{p}>.05$ ). $P$. sungorus pulled less cotton than all but one of the eight species previously tested under these conditions.

For nest type, the mean nest rating for each individual was used as the basic datum. The median nest rating for males was 2.25, and that for females, 2.11. There was not a significant sex difference (Mann-Whitney $U=26, p>$ $.05)$. During the 14-day period of data collection, there was only one instance in which no nest was built; 14 platform nests, 178 cup-shaped nests, and 87 covered nests were built. The nest rating scores of $P$. sungorus ranked in the middle of the eight previously tested species.

\section{EXPERIMENT 10}

Parental care has long been a topic of interest among animal behaviorists. Recently, various authors have attempted to relate patterns of parental care to mating systems and to the relative influences of $r$ and $K$ selection. Hartung and Dewsbury (1979b) compared the parental behavior of several species of muroid rodents in a standardized laboratory setting in an effort to evaluate such hypotheses. Experiment 10 examined the parental behavior of $P$. sungorus.

\section{Method}

Ten male-female pairs of $P$. sungorus served as subjects for this experiment. Adult (65-200 days) pairs were placed together in $48 \times 27$ $\times 13 \mathrm{~cm}$ clear plastic cages with San-i-cel as substrate and were checked daily for the presence of litters. Each cage contained a cotton pad (Nestlet, Ancare Corp.) as nesting material. Behavioral observations of parental care were conducted on the first litter produced by each pair. Large litters were culled to three pups; all litters contained at least two pups. Tests were conducted during the middle of the dark phase with illumination provided by a $25-\mathrm{W}$ red bulb suspended over the home cage. Data were recorded on Days 2, 3, 9, and 10 after birth.

Each test lasted $1 \mathrm{~h}$ and was divided into two parts. During the first $30 \mathrm{~min}$, the behavior of both parents was recorded (paired condition). Then one parent was removed and, after a 15-min adaptation period, the behavior of the remaining parent was recorded for an additional $30 \mathrm{~min}$ (single condition). Each parent was tested twice in the single condition on either Days 2 and 9 or Days 3 and 10. The order of testing was counterbalanced. The following behaviors were recorded for each parent (when present): sitting on nest, manipulating pups, manipulating nest material, and pup retrieval. These categories correspond to those used by Hartung and Dewsbury (1979b), with one exception; in the present study, licking and manipulating pups were both recorded in the category of manipulating pups.

\section{Results and Discussion}

Except for nursing the pups, paternal care and maternal care were qualitatively similar in $P$. sungorus. In the 
Table 6

Results of Study of Parental Behavior in Phodopus sungorus

\begin{tabular}{lccc} 
Condition & $\begin{array}{c}\text { M Duration } \\
\text { Sitting on } \\
\text { Nest }\end{array}$ & $\begin{array}{c}\text { Manipulating } \\
\text { Pups }\end{array}$ & $\begin{array}{c}\text { Manipulating } \\
\text { Nest Materials }\end{array}$ \\
Female & \multicolumn{2}{c}{ Paired } & \\
Male & 899.1 & 56.3 & 57.6 \\
& 192.9 & 15.5 & 14.7 \\
Female & Single & & \\
Male & 895.1 & 55.9 & 69.9 \\
\hline
\end{tabular}

Note-Durations are expressed in seconds.

paired condition, males spent less time on the nest (Wilcoxon $\mathrm{T}=0, \mathrm{p}<.01$ ) and less time manipulating pups $(T=0, p<.01)$ or nesting materials $(T=2, p$ $<$.01) than did females. Females displayed these three behaviors for approximately equal durations, regardless of whether they were the single parent present or paired with a male. Males spent more time on the nest when the female was absent than when she was present $(T=4, p$ $<.02$ ) (see Table 6). Females retrieved pups a total of 64 times with a male present and only 13 times when alone. Males retrieved pups on 3 occasions in each condition.

The durations spent on the nest by males and females when each was the single parent were lower than the durations for all but one of the previous six species investigated.

\section{GENERAL DISCUSSION}

To construct the "adaptive profile" for $P$. sungorus, one or two representative measures from each experiment were selected for inclusion (Dewsbury et al., 1982). The resulting 14 measures for this study were each plotted on an axis, the range of which was defined by the lowest and highest scores of all species thus far tested (Figure 3). For each of the 14 scales, we have data from previous studies in this laboratory for a range of from 6 (parental behavior) to 42 (copulatory behavior) species. Each of the 14 species for which we have data for at least seven of the categories has been assigned a number and is included in Figure 3 .

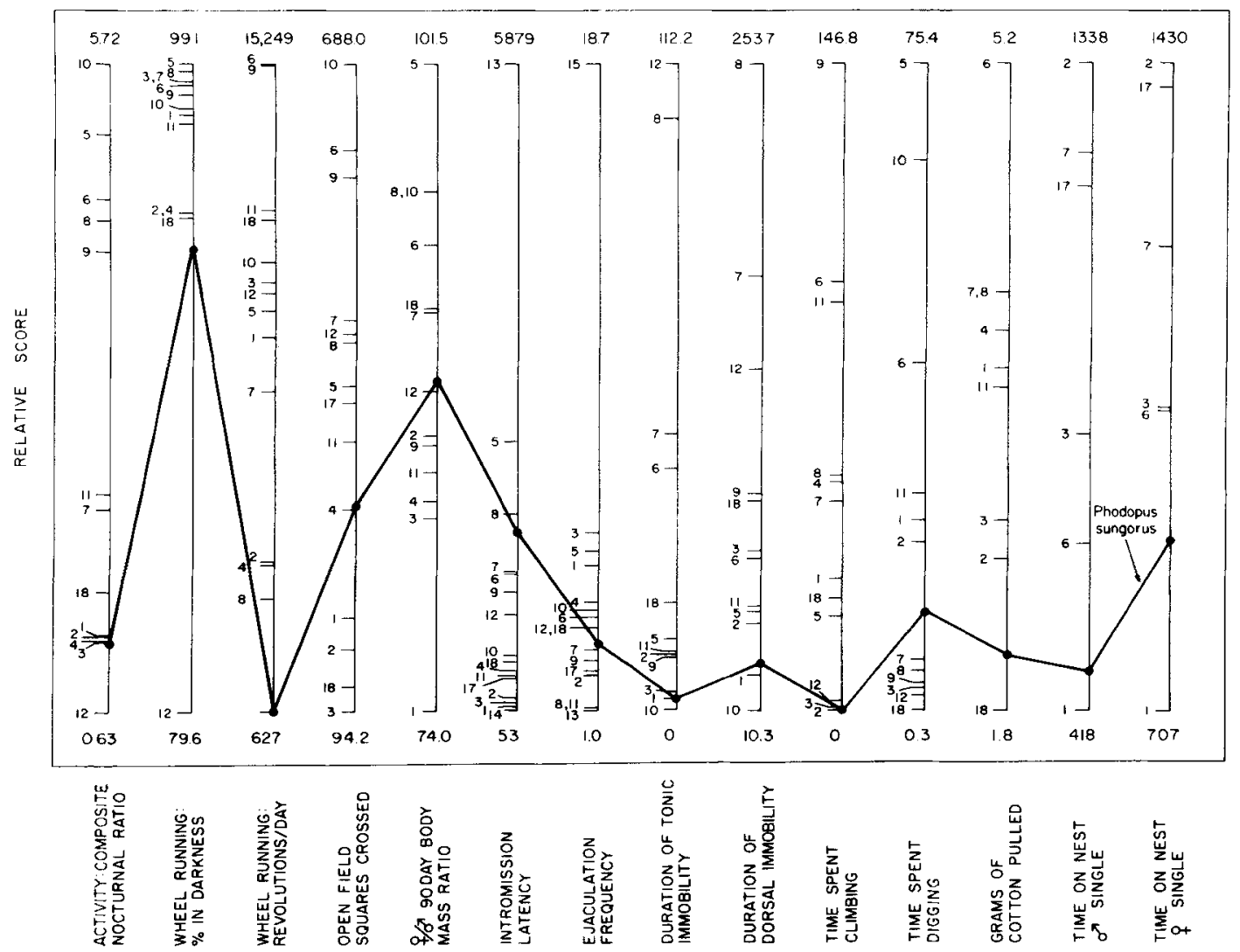

Figure 3. The adaptive profile. The 14 scales shown relate to studies of 10 behavioral categories in this laboratory. Scores for Phodopus sungorus are indicated by a solid line. The values for other species for which data are available on at least 7 of 10 behavioral categories are represented also: (1) Microtus montanus, (2) $M$. ochrogaster (3) $M$. pennsylvanicus, (4) M. canicaudus, (5) Peromyscus polionotus, (6) $P$. maniculatus bairdi, (7) $P$. leucopus, (8) $P$. eremicus, (9) P. gossypinus, (10) Onychomys leucogaster, (11) Mus musculus, (12) Rhabdomys pumilio, and (18) Clethrionomys gapperi. Where their scores are the most extreme among species studied, values are also shown for: (13) Baiomys taylori, (14) Rattus norvegicus, and (15) Onychomys torridus. In addition, values on five categories are shown for Microtus californicus (17). 
The scores for $P$. sungorus are connected by a solid line to permit rapid evaluation. The data for any of the other 14 species can be similarly connected for comparison. In a few instances, the most extreme scores on a scale were from species other than these 14 species or P. sungorus, and these data are also included.

By comparing behavioral data collected in the laboratory from numerous closely related species, it is hoped that insight can be gained as to the relative importance and frequency of certain behaviors to various species in their natural habitats. Additionally, clusters of behavioral adaptations may appear repeatedly in certain groups and thereby lead to testable hypotheses as to the variables that influence social and mating systems as well as total life history strategies. In conjunction with various physiological measures (e.g., metabolic rates, hormonal levels), a more detailed understanding of the proximate and ultimate influences on behavior may emerge.

The first four scales of the adaptive profile represent experimental situations that have been used by various authors as measures of activity. When observed throughout a 24-h cycle, the behavior of $P$. sungorus is distributed acyclically. That is, there is no rhythmicity in their behavior when analyzed on an hour-by-hour basis (see Figure 2). As can be seen on the adaptive profile, this acyclic distribution of behavior is very similar to that of the four Microtus species previously investigated. Baumgardner et al. (1980) suggested that the acyclic pattern shown by Microtus species might reflect their relatively cold environments and resulting high metabolisms and high nutritional requirements. In the present study, $P$. sungorus showed relatively high levels of eating equally distributed across the 24-h cycle, suggestive of a similar relationship.

The comparatively low percentage of wheel revolutions made in the dark by the hamsters seems to agree well with the above findings. Most species previously investigated are more nocturnal in their distribution of wheel running. However, in this apparatus, $P$. sungorus are at least somewhat nocturnal, although this trend is due virtually entirely to the behavior of three markedly nocturnal animals (see Figure 2). More pronounced nocturnality in the running wheels, when compared with home cage activity, was also found for Microtus species (Dewsbury et al., 1982).

Previous reports about the daily patterning of the behavior of these animals in the field are rare. Occasionally they are described as nocturnal, and at least one author (Kontschinz, 1956) suggests that although they are most active at night, they are also active during the day. In addition, $P$. sungorus typically inhabit covered areas entangled by vegetation (Meyer, 1967) and are reported to frequent the runway systems of pikas (Loukashkin, 1940), both habits that might be conducive to diurnal or acyclic activity.

The mean number of daily revolutions in running wheels made by $P$. sungorus was the lowest yet observed in this laboratory. Even the three most active animals made fewer revolutions than the lowest mean previously recorded. Very low levels of locomotion (along with exceptionally high levels of sleep) were also recorded in Experiment 1. If, indeed, the distribution of locomotor-exploratory behavior in these animals is related to their high caloric requirements, then their relatively low levels of locomotion in these experimental settings may relate to the ad-lib availability of food. Increased levels of locomotion might emerge in the presence of a more sparsely distributed food source.

Taken together, the amount of locomotor activity shown by $P$. sungorus in Experiment 1 (24-h activity patterning), Experiment 2 (wheel running), and Experiment 3 (open field) was low and similar to that shown by Microtus. Also, the relatively acyclic patterning of locomotion shown by Microtus appeared in Experiments 1 and 2. In general, the levels of locomotion shown by $P$. sungorus are more like those shown by various Microtus species than those of other species investigated.

There are previous reports indicating that $P$. sungorus males are heavier than females (Emmerick, 1978a, 1978b; Figala, Hoffman, \& Goldau, 1973). Several authors suggest that sexual dimorphism for body mass will be reduced in monogamous species relative to that in species with polygamous or promiscuous breeding systems (Dewsbury et al., 1980; Kleiman, 1977). Because the data from this study were collected using a single population and utilizing uniform maintenance conditions, many of the biases inherent in field dimorphism data, including seasonal mass fluctuations, condition, and nutrition, were avoided. The dimorphism found in this study is intermediate compared to that for other species studied. Nevertheless, the fact that males were significantly heavier than females suggests an absence of monogamy in this species.

Dewsbury (1981) has also proposed that long latencies to initiate copulation may be correlated with monogamous mating systems. As choice of a mate by a monogamous animal may have greater effects on lifetime fitness than a similar choice by a polygamous animal, Dewsbury (1981) has suggested that courtship patterns of monogamous species should be relatively complex. This complexity may be correlated with long latencies to initiate copulation, as is seen in some species for which evidence from other sources also suggests monogamy (e.g., Peromyscus polionotus, Dewsbury, 1971). The intromission latencies found for $P$. sungorus in Experiment 5 are comparatively long $(1894.7 \mathrm{sec})$, being exceeded only by the latencies of 5 of 42 previously investigated species. However, in 20 additional tests using females that were in cycling estrus and housed across a wire-mesh barrier from the male, the mean intromission latency was $682.3 \mathrm{sec}$, making the relation of this measure to mating system difficult to interpret. Since both proximity to the male and mode of estrus differed from the conditions in Experiment 5, a single factor responsible for these reduced intromission latencies cannot be identified with any certainty.

In addition to reduced sexual dimorphism and long intromission latencies, Dewsbury (1981) has assembled seven other behavioral and morphological variables that have been proposed as correlates of monogamy. The monogamy scale requires validation but is presented as a heuris- 
Table 7

Scores of Phodopus sungorus on Monogamy Scale of Dewsbury (1981)

\begin{tabular}{lcc}
\hline \multicolumn{1}{c}{ Behavioral Pattern } & Score & $\begin{array}{c}\text { Scale } \\
\text { Value }\end{array}$ \\
\hline 1. Dimorphism & yes & -2 \\
2. Intromission Latency & $1895 \mathrm{sec}$ & +2 \\
3. Allogrooming & & \\
4. No. of Ejaculations & 2.9 & 0 \\
5. Coolidge Effect & & \\
6. Plug & yes & -1 \\
7. Litter Size* & 3.6 & 0 \\
8. Paternal Behavior & yes & +1 \\
9. Eyes Open & 11.6 days & -1 \\
$\quad$ Sum Scale Score & & -1 \\
\hline
\end{tabular}

*Data from Jordan (1971)

tic device and generates values that are generally congruent with what is known about mating systems in these species (Dewsbury, 1981). Each species was assigned a value (ranging from +2 to -2 ) for each variable, depending on where it scored in relation to the other species. A total score was then calculated (see Table 7). The scores of the 21 species for which there are data for at least six of the nine categories range from +10 to -12 , with the probability of monogamy presumably correlated with increasing positive scores. Data are available for seven of the nine variables for $P$. sungorus. The cumulative score for the hamsters $(-1)$ suggests that monogamy is not likely to be their most frequently occurring breeding system.

The single thrust with each intromission (see below) and prerequisite multiple insertions preceding ejaculation shown by $P$. sungorus are similar to the pattern of Mesocricetus auratus. An additional similarity involves the occasional intromission, virtually always in later ejaculatory series, that includes more than one thrust. These similarities presumably reflect both species' classification as true hamsters.

Regardless of whether or not this species actually forms a lock at ejaculation, the copulating pair is presumably at high risk to predation during a fairly long period (approximately $10 \mathrm{sec}$ ) when they lie on their sides joined together. That several species with similar copulatory patterns have much shorter ejaculation durations suggests a possible response to dense plant cover (Dewsbury, 1978).

As reported by Emmerick (1978a, 1978b), males deposit a copulatory plug at ejaculation. Dewsbury (1978) has suggested that in locking species the lock may serve some of the presumed functions (Voss, 1979) of a plug and that locking species, which typically have a reduced complement of male accessory glands, will not deposit plugs. If indeed $P$. sungorus do lock, they would be the first reported species to have both a copulatory lock and deposit copulatory plugs.

As for virtually all other species studied, the dorsal immobility procedure was more effective than the tonic immobility procedure. It has been suggested that the tonic immobility response is related to a predator response strategy (Gallup, 1977; Webster, Lanthorn, et al., 1981).
The protection afforded $P$. sungorus by dense vegetation may have reduced the tendency to become immobile in the presence of a predator, as hypothesized for Microtus by Webster, Lanthorn, et al (1981). It should also be noted that the only individual that displayed the tonic immobility response in the present study vocalized continuously while being handled and appeared to be highly aroused. The present testing situation may not have provided sufficient stimulation to induce the response in these extremely docile animals.

That the hamsters did not climb is not surprising in light of both their anatomy and habitat. Their stocky bodies and short tails and legs all suggest a terrestrial or semifossorial existence (Horner, 1954). Flint (1966) reported that these and closely related hamsters are not found in forest areas.

All hamsters did some digging on the sand substrate, and 7 of $10 \mathrm{dug}$ on peat. The total time spent digging, however, was not exceptional. As reported by Emmerick (1978a, 1978b), $P$. sungorus often dig briefly at the surface and then proceed to roll vigorously, presumably scent marking the area. The time spent digging by $P$. sungorus was very close to the mean for the four Microtus species previously tested.

Since the steppe regions inhabited by this species are characterized by relatively cold winters, it is at first surprising that the hamsters pulled so little cotton. However, the thick coats of the animals and the relatively high temperatures in the laboratory may have combined to reduce the need for the insulation provided by the cotton.

As also reported by Gibber, Piontekewitz, and Terkel (1978), we found male parental care to be similar to but less frequent than maternal care. This finding is typical of muroid rodents in the laboratory (Hartung \& Dewsbury, 1979b).

Phodopus sungorus has not been the subject of intense field study with respect to behavior. The adaptive profile adds significantly to our understanding of this species by corroborating many of the anecdotal descriptions of field behavior that have appeared in the literature. The profile also provides additional information as to the adaptations of $\boldsymbol{P}$. sungorus to the environment, as well as some hints as to the social system of the species.

When the entire adaptive profile is examined (see Figure 3), several interesting comparisons with previous species can be made. Dewsbury et al. (1982) have recently reported means for two genera for which several data points exist for several of the same scales under consideration in the present study. The means reported for four Microtus species on the adaptive profile reveal a cluster of scores that bear striking similarities to those for $P$. sungorus. Microtus species and $P$. sungorus show very similar patterns and levels of activity. Both distribute home cage behavior acyclically throughout the 24-h period. Microtus and $P$. sungorus both are less nocturnal in their distribution of wheel running than virtually all species previously investigated, and both make relatively few revolutions per day and few square crossings in the open field. Phodopus sungorus displayed low durations of both immobility responses, again similar to those for Microtus. 
Durations of digging and climbing were also similar for the hamsters and Microtus species. Microtus generally climbed very little, whereas Phodopus did not climb at all.

These rather striking behavioral similarities in the laboratory are correlated with several additional factors. Both $P$. sungorus and Microtus species inhabit areas where severe winters and warm summers are typical. Also, both frequent habitats with at least fairly dense plant cover. There are also similarities in the appearance of $P$. sungorus and Microtus. They are all relatively squat, with short legs and tails.

Considered together, these behavioral, environmental, and physical similarities suggest that $P$. sungorus may have evolved a cluster of behavioral adaptations that are, at least in part, a response to environmental influences that are similar to those shared by several North American Microtus species. Additional information regarding Phodopus behavior in the field would provide tests of several hypotheses. Are there cyclic population crashes among $P$. sungorus as there are in many Microtus species? What relationships exist between $P$. sungorus and other central Asian rodent species? It is hoped that the adaptive profile will provide a starting point for many other similar questions.

\section{REFERENCES}

Altmann, J. A. (1974). Observational study of behavior: Sampling methods. Behaviour, 49, 227-267.

Baumgardner, D. J., WArd, S. E., \& Dewsbury, D. A. (1980). Diurnal patterning of eight activities in 14 species of muroid rodents. Animal Learning \& Behavior, 8, 322-330.

DALY, M. (1976). Behavioral development in three hamster species. Developmental Psychology, 9, 315-323.

DewsBuRY, D. A. (1971). Copulatory behavior of old-field mice (Peromyscus polionotus subgriseus). Animal Behaviour, 19, 192-204.

DEwSBURY, D. A. (1975). Diversity and adaptation in rodent copulatory behavior. Science, 190, 947-954.

DEWSBURY, D. A. (1978). The comparative method in studies of reproductive behavior. In T. E. McGill, D. A. Dewsbury, \& B. D. Sachs (Eds.), Sex and behavior: Status and prospectus. New York: Plenum Press.

DewsBURY, D. A. (1980). Wheel-running behavior in 12 species of muroid rodents. Behavioural Processes, 5, 271-280.

DewsBury, D. A. (1981). An exercise in the prediction of monogamy in the field from laboratory data on 42 species of muroid rodents. The Biologist, 63, 138-162.

Dewsbury, D. A., Baumgardner, D. J., Evans, R. L., \& Webster, D. G. (1980). Sexual dimorphism for body mass in 13 taxa of muroid rodents under laboratory conditions. Joumal of Mammalogy, 61, 146-149.

Dewsbury, D. A., Baumgardner, D. J., SaWrey, D. K., Webster, D. G. (1982). The adaptive profile: Comparative psychology of redbacked voles (Clethrionomys gapperi). Joumal of Comparative and Physiological Psychology, 96, 649-660.

DewsBuRY, D. A., DAWson, W. W. (1979). African four-striped grass mice (Rhabdomys pumilio), a diurnal-crepuscular rodent, in the behavioral laboratory. Behavior Research Methods \& Instrumentation, 11, 329-333.

Dewsbury, D. A., Lanier, D. L., \& Miglietta, A. (1980). A laboratory study of climbing in 11 species of muroid rodents. American Midland Naturalist, 103, 66-72.

EMmerick, J. J. (1978a, March). Behavioral patterns of the djungarian hamster (Phodopus sungorus). Paper presented at the meeting of the Midwestern Animal Behavior Society, West Lafayette, IN.
EMMERick, J. J. (1978b, June). Mating behavior of djungarian hamsters. Paper presented at the meeting of the Eastern Conference on Reproductive Behavior, Madison, WI.

Figala, J., HoffmanN, K., \& Goldau, G. (1973). Zur Jahresperiodik beim Dsungarischen Zwerghamster Phodopus sungorus. Oecologia, 12, 89-118.

FuINT, W. E. (1966). Die Zwerghamster der palaarktischen Fauna. Wittenberg: Ziemsen.

GalluP, G. G., JR. (1977). Tonic immobility: The role of fear and predation. Psychological Record, 27, 41-61.

Gibber, J. R., Pointekewitz, Y., \& Terkel, J. (1978, June). Maternal and paternal behavior of Siberian hamsters (Phodopus sungorus). Paper presented at the meeting of the Animal Behaviour Society, Duluth, MN.

Hartung, T. G., Dewsbury, D. A. (1979a). Nest building behavior in seven species of muroid rodents. Behavioral Biology, 27, 532-539.

Hartung, T. G., Dewsbury, D. A. (1979b). Paternal behavior in six species of muroid rodents. Behavioral Biology, 26, 466-478.

hatton, D. C., Lanthorn, T., Webster, D. G., \& Meyer, M. E. (1978). Baroreceptor involvement in the immobility reflex. Behavioral Biology, 22, 122-127.

HoffmanN, K. (1982). The effect of brief light pulses on the photoperiodic reaction in the djungarian hamster (Phodopus sungorus). Journal of Comparative Physiology, 148, 529-534.

HoRner, B. E. (1954). Arboreal adaptations of Peromyscus with special reference to use of the tail. Contributions from the Laboratory of Ver tebrate Biology of the University of Michigan, 61, 1-84.

Jarman, P. J. (1982). Prospects of interspecific comparison in sociobiology. In Kings College Sociobiology Group (Eds.), Current problems in sociobiology. New York: Cambridge University Press.

JORDAN, J. (1971). The establishment of a colony of djungarian hamsters (Phodopus sungorus) in the United Kingdom. Journal of the Institute of Animal Technicians, 22, 56-61.

Kleiman, D. G. (1977). Monogamy in mammals. Quarterly Review of Biology, 52, 39-69.

KLEMM, W. R. (1971). Neurophysiological studies of the immobility reflex (animal hypnosis) In S. Ehrenpresis \& O. C. Solnitzky (Eds.), Neuroscience Research (Vol. 4, pp. 165-212). New York: Academic Press.

KonTSCHINZ, J. (1956). Rost, yazvitie i razmnoshenie dsungarkich chomjakov [Growth, development and reproduction of dwarf djungarian hamsters]. Moskovskii Zoopark; sbornik statei, 1, 66-75.

LoukASHKIN, A. S. (1940). On the pikas of North Manchuria. Journal of Mammalogy, 21, 402-405.

MEYER, M. N. (1967). [Peculiarities of the reproduction and development of Phodopus sungorus Pallas of different geographical populations] (in Russian). Zoologicheskii Zhurnal, 47, 604-614.

RALLS, K. (1976). Mammals in which females are larger than males. Quarterly Review of Biology, 51, 245-276.

Vorontsov, N. N., RADZhABLI, S. I., \& Lyapunova, K. L. (1967). Karyological differentiation of allopatric forms of hamsters of the superspecies Phodopus sungorus and heteromorphism of the sex chromosomes in the females. Doklady Akademi Nauk SSSR, 172, 703-705.

Voss, R. (1979). Male accessory glands and the evolution of copulatory plugs in rodents. Occasional papers of the Museum of Zoology, University of Michigan, 689, 1-17.

Webster, D. G., BAumgardner, D. J., \& Dewsbury, D. A. (1979). Openfield behavior in eight taxa of muroid rodents. Bulletin of the Psychonomic Society, 13, 90-92.

Webster, D. G., Lanthorn, T. H., Dewsbury, D. A., Meyer, M. E. (1981). Tonic immobility and the dorsal immobility response in twelve species of muroid rodents. Behavioral and Neural Biology, 31, $32-41$.

Webster, D. G., Williams, M. H., OWens, R. D., Geiger, V. B., \& DewsBuRY, D. A. (1981). Digging behavior in 12 taxa of muroid ro dents. Animal Learning \& Behavior, 9, 173-177.

Wilson, R. C., VaCeK, T., LANier, D. L., Dewsbury, D. A. (1976). Open-field behavior in muroid rodents. Behavional Biology, 17, 495-506.

(Manuscript received January 24, 1984; revision accepted for publication May 25,1984 .) 